\title{
Contenido de metales pesados en agua y sedimento en el bajo Nanay
}

\author{
[Heavy metals content in water and sediment in low Nanay]
}

\author{
Víctor Sotero-Solís ${ }^{1 *} \&$ Mario Alva-Astudillo² \\ ${ }^{1}$ Instituto de Investigaciones de la Amazonia Peruana. Carretera Iquitos-Nauta Km 4,5-Iquitos. \\ ${ }^{2}$ Facultad de Ingeniería Química. Universidad Nacional de Trujillo. Ciudad Universitaria. Juan Pablo II s/n., Trujillo, Perú. \\ *e-mail: proyectopalmeras@gmail.com
}

\begin{abstract}
Resumen
Se presenta en este estudio la evaluación de la concentración de metales pesados en agua y mercurio en sedimentos del rio Nanay, s realizó el muestreo desde el caserío Libertad, hasta su desembocadura en el rio Amazonas, en las épocas de creciente y vaciante del año 2011. La cuenca del rio Nanay comprende $340 \mathrm{~km}^{2}$ s y alberga a cuatro comunidades nativas y ocho campesinas y además tiene influencia sobre los distritos de San Juan, Iquitos y Punchana, además de ser la fuente de agua potable para la población de estos distritos. Los análisis de metales pesados se realizarán utilizando la técnica de la absorción atómica en el laboratorio de Sustancias Naturales Bioactivas del Instituto de Investigaciones de la Amazonia Peruana (IIAP). Según los resultados de añiláis de agua el plomo y mercurio se encuentran presentes sen concentraciones mayores que lo indicado por las normas nacionales. El plomo tanto en creciente es en promedio de 0,111 ppm y $0,053 \mathrm{ppm}$ respectivamente y el mercurio en vaciante se encuentra en 0,008 ppm Del mismo modo la presencia de mercurio es alta en los sedimentos que acompañan a este rio con 1,636 ppm en creciente y 3,03 ppm en vaciante.
\end{abstract}

Palabras clave: metales pesados, ríos amazónicos, contaminación.

\section{Abstract}

It is presented in this study the evaluation of the concentration of heavy metals in water and mercury in sediments of the Nanay river, carried out the sampling since the population of Liberty, to its estuary in the Amazon river, in the flood season and dry season of 2011. The basin of the Nanay river, is of $340 \mathrm{~km} 2 \mathrm{~s}$ and host to four native communities and eight peasants and besides has influence on the districts of San Juan, Iquitos and Punchana, besides being the source of drinking water for the population of these districts. The analyses of heavy metals were carried out utilizing the technique of the atomic absorption in the laboratory of Bioactive Natural Substances of the Institute of Investigations of the Amazonia Peruvian (IIAP). According to the results of the water, the leads and mercury presents are found with concentrations higher than it indicated by the national norms. The lead as in food season as in dry season is on the average of $0.111 \mathrm{ppm}$ and $0.053 \mathrm{ppm}$ respectively and the mercury in dry season is found in $0.008 \mathrm{ppm}$ in the same way the presence of mercury is high in the sediments that accompany to this river, with $1.636 \mathrm{ppm}$ in food season and $3.03 \mathrm{ppm}$ in dry season.

Keywords: heavy metals, Amazonian rivers, contamination. 


\section{INTRODUCCIÓN}

La contaminación de los ríos de la amazonía peruana ha devenido en un serio problema, sobre todo cuando en su cuenca se encuentran productos valiosos como son la madera, petróleo, oro y producción de cocaína cuya extracción legal o ilegal está causando un serio deterioro al ecosistema amazónico. Los ríos más afectados severamente por las actividades antropomórficas en la amazonia, son los tributarios del Marañón, Huallaga, Madre de Dios, Napo y Nanay, entre otros, y los resultados de este deterioro se reflejan en problemas en la flora, fauna y salud de los pobladores de esta región (Gómez, 1995; Álvarez et al., 2011)

El río Nanay, es afluente del río Amazonas y forma la cuenca del mismo nombre, la cual tiene una extensión de 1,721, 343 has; recorre los distritos de Alto Nanay, San Juan, Iquitos y Punchana. Es un río considerado estratégico, porque el abastecimiento de agua para consumo humano, a la ciudad de Iquitos y distritos aledaños. Es un rio de origen meandrito con una longitud de $442 \mathrm{~km}$. El lecho del río está conformado por material arenoso con cantos rodados y limo, en su recorrido se pueden observar una gran cantidad de playas constituidas de arena blanca. (IIAP, 2002)

Según reportes de Capitanía de Puertos de Iquitos en el año 1999 ingresan las primeras dragas ilegalmente a la cuenca del río Nanay, con el pretexto de extraer material de acarreo (gravilla y arena), al año siguiente se intensifica este tipo de actividad con el incremento de más dragas, crece entre la población el rumor de que las mismas extraían oro, en efecto el interés creciente de otras empresas extiende esta ilícita actividad hacia el Pintuyacu y el Alto Nanay. La mayoría de estas dragas no contaban con ningún tipo de autorización, en muchos casos no contaban incluso ni con el permiso de zarpada que otorga Capitanía de Puertos o de lo contrario la zarpada otorgado para un destino era utilizado para otro distinto al autorizado. Para entonces se realiza el primer muestreo de aguas y se comprueba que los índices de Mercurio se encontraban aún dentro de los parámetros normales. En el año 2001 se intensifica la actividad minera, así como el número de dragas operando bajo la simple modalidad de extracción de material de acarreo únicamente.

Nuevamente se realiza un monitoreo de la calidad de aguas y sedimentos y se determina presencia creciente de niveles de Mercurio $\mathrm{Hg}$. En las aguas y peces en el mes de mayo. En octubre se toma muestras entre aguas, sedimentos, peces y muestras de sangre, cabello y orina de personas de poblaciones aledañas a las dragas, dichos resultados no fueron difundidos por DIGESA, sino hasta junio del año 2002, perjudicando así a los afectados, que a la fecha no reciben ningún tipo de tratamiento (Barreto, 2003).

El IIAP (2008), realizó análisis de los efluentes del botadero municipal y que afectaban la quebrada Santa Cruz que cruza la reserva Allpahuayo-Mishana y posterior desembocadura en el río Nanay, encontrándose concentraciones altas de mercurio y plomo, muy por encima de los limites permitidos por Los Estándares nacionales de calidad ambiental.

El suelo es removido constantemente de la superficie de la tierra y transportado aguas abajo por los ríos hasta que se deposita finalmente en los lagos, estuarios y océanos., considerándose así al agua como el principal88 agente de la erosión y el vehículo principal del material erodado. Los sedimentos se mueven en un cauce natural como "sedimento en suspensión" en el flujo o como "carga de fondo", que se desliza y rueda a lo largo del lecho del canal. Los procesos no son independientes entre si, dado que el material que aparece en un tramo como carga de fon do puede aparecer aguas abajo en suspensión. La producción de sedimentos de una cuenca depende de muchos factores tales como el clima, el tipo de suelos, el uso de la tierra, la topografía y la existencia de embalses y está determinada por la unidad de área con la precipitación media anual. De acuerdo al análisis de numerosas cuencas, la tasa de producción máxima ocurre para aproximadamente $305 \mathrm{~mm}$ de precipitación media anual, dado que en esas condiciones existe poca cobertura vegetal. Con precipitación mas intensa, la vegetación prolifera y reduce la erosión y con lluvias 
bajas también ocurre una reducción (Linsley et al., 1994).

Los metales pesados que son introducidos en un sistema acuático, generalmente son depositados a través de procesos de precipitación y sedimentación en la capa de sedimento (Avila-Pérez et al., 1999; Rodríguez y Avila-Pérez, 1997). De tal manera que el sedimento provee información sumamente valiosa de la historia de la calidad del cuerpo de agua (Baryshev et al., 1995; Von Gunten et al., 1997).

Es sabido que los metales ligados a los sedimentos a concentraciones suficientes ejercen efectos adversos sobre la biota residente (Brannon et al., 1980; Burgess y Scott, 1992). Algunos metales pesados pueden ser absorbidos en altas concentraciones por la vegetación. El mercurio, cobre, níquel, plomo, cobalto, cadmio, entre otros, son los metales que se muestran como los más tóxicos en concentraciones excesivas para las plantas superiores (Alloway, 1995), así como para otros organismos. Esta toxicidad también puede afectar a otros niveles superiores de la cadena alimenticia, tanto por ingesta directa como por la potencial transmisión a través de la red trófica estuarina (Adam, 1990). Según Lopez (2009), para que cualquier compuesto sea absorbido por una planta, éste debe de estar en contacto con su raíz y por diversos mecanismos pasan a la raíz, pudieno ser por flujo de masa, difusión o impercepción con la misma.

Según Valdés y Cabrera (1999), los metales pesados se han convertido en un tema actual tanto en el campo ambiental, como en el de salud pública. Los daños que causan son tan severos y en ocasiones tan ausentes de síntomas, que las autoridades ambientales y de salud de todo el mundo ponen mucha atención en minimizar la exposición de la población, en particular de la población infantil, a estos elementos tóxicos.

La contaminación por metales pesados en los cuerpos y sedimentos de agua ha sido un tema de investigación bastante amplio, sobre todo los que se originan debido a las cercanías de relaves mineros, perforaciones, producción y refinación de petróleo y los procedentes de los desechos municipales. Así se tiene los reportados por Labunska et al., (2000) en las aguas alrededor de la refinería de YPF en Ensenada, Buenos Aires (Argentina), con alta concentración de zinc, mercurio y cobre. En México, García et al. (2007), reportan presencia de cobre, zinc y plomo, aunque en cantidades moderadas en el embalse Alzate. Sánchez et al., (2000), encontraron concentraciones altas de cromo y plomo en el rio San Javier, procedente de las aguas del sistema general de desagüe $y$ drenaje de la ciudad de México, Janiot et al., (2001), realizaron análisis del agua y sedimentos en el área de descarga del rio de la Plata, encontrando alta contaminación de todos los metales y sobre todo de cadmio en esta área. Monroy et al., (2002) encontraron que el arsénico excede en cinco veces el limite permisible $(50 \mu \mathrm{g} / \mathrm{L})$ en los tanques de almacenamiento de Villa Paz y Matehuela en San Luis de Potosí, conocido centro minero. Rojas (1988), realiza un trabajo de análisis de metales pesados del río Rímac en Casapalca y Parac, observando alta concentración de plomo.

El objetivo del presente estudio es determinar la concentración de metales pesados en el agua y suelo inundable de la cuenca del río Nanay, en la parte que circunda a los distritos de San Juan, Iquitos y Punchana.

\section{MATERIALES Y MÉTODOS \\ Material}

Se realizó la colecta de las muestras de aguas superficiales del rio Nanay desde la localidad de Libertad, en el límite de la Reserva Allpahuayo Mishana hasta la desembocadura de este rio en el Amazonas, en Bellavista Nanay. Los puntos de muestreo serán: Libertad- Nina Rumi- Fuente de captación de agua potable- Moronococha - Bellavista.

\section{Métodos}

Los análisis de $\mathrm{pH}$ y temperatura fueron tomadas en el lugar de muestreo.

Para metales pesados para agua, se realizaron por espectrofotometría de absorción atómica en horno de flama para determinar plomo, arsénico, cadmio y cromo (Caldas, 2004). Por otra parte, la determinación de mercurio se realizará mediante la espectrofotometría de 
absorción atómica en vapor frío (EAA-VF), por ser una técnica de una alta sensitividad y sin interferencias espectrales (Norma Oficial Mexicana117-1994, Jorheim, 2001)

\section{RESULTADOS Y DISCUSIÓN}

La localización de los puntos según las coordenadas dadas en la Tabla 2, fueron desde el caserío Libertad, colindante con la reserva Allpahuayo-Mishana hasta la desembocadura del río Nanay en el Amazonas, cerca al caserío Bellavista en el distrito de Punchana; equivale en línea recta la cantidad de $22,6 \mathrm{Km}$.

De acuerdo a la Tabla 3, donde se analizan las muestras de agua del rio Nanay, en creciente, y analizando los resultados con los límites máximos permisibles nacionales (Tabla1), se observa que, en hierro, todos pasan para la categoría 1, para la categoría 3, solo sobrepasan las muestras posteriores al caserío Libertad y Morona. El zinc no sobrepasa a los límites en ninguna de las categorías. El manganeso, solo la muestra que pasa por Morona sobrepasa los límites y en plomo se sobrepasan en todas las categorías. En mercurio solo la muestra del punto 05, sobrepasa los límites dados para todas las categorías.

Asimismo, en la Tabla 3, se puede apreciar un incremento de los metales cobre, fierro, magnesio, zinc, manganeso y plomo después de pasar por la ciudad de Iquitos. Se debe de considerar que esta zona es la que recibe los mayores colectores de desagüe de la ciudad de Iquitos.

De acuerdo a la Tabla 4, donde se analizan las muestras de agua colectadas en la vaciante del río Nanay, y comparando estos valores con los límites máximos permisibles nacionales, para el cobre, ninguna de las muestras sobrepasa los límites indicados, en hierro todas las muestras sobrepasan en la categoría 1 y 3, excepto en la muestra de Bellavista. En cuanto al magnesio, ninguna muestra sobrepasa la categoría 3 , que es la única que dan las normas nacionales, Ninguna sobrepasa al elemento zinc, lo mismo sucede con el manganeso (no hay en la categoría 4). En plomo las muestras sobrepasan en todas las categorías, con excepción de la muestra de $200 \mathrm{~m}$ antes del caserío Libertad, En cadmio en todos los puntos no sobrepasan los límites máximos y en mercurio sobrepasan en todas las categorías.

Del mismo modo en la Tabla 4, se puede observar que todos los metales pesados sufren un incremento notable, sobre todo cuando pasan la parte que corresponde a la ciudad de Iquitos, incrementándose en el Punto 5 (Munich) y Bellavista. El plomo y manganeso no se incrementaron en Munich.

Comparando los resultados de las tablas 3 y 4 en promedio, se puede apreciar que, de acuerdo a los promedios de creciente $y$ vaciante, permanecen idénticas los elementos cobre y cadmio; se incrementan hiero y mercurio y disminuyen magnesio, zinc $y$ manganeso.

Observando la Tabla 5 y Tabla 6, se observa que las concentraciones de cobre y zinc difieren estadísticamente en todos los puntos. Salvo el cadmio, las mayores acumulaciones se presentan en el sector de Pampa Chica. Se debe de tener en consideración la alta concentración de hierro en los sedimentos del río Nanay, este elemento varia de 857,68 a 3241,1 ppm, en los puntos de Bellavista en creciente y abajo Libertad en vaciante. Observando la Tabla 5 en cuanto al mercurio, este se encuentra en el rango de 0,015 a 0,075 ppm y en la Tabla 6, el rango varia de 0,33 a 0,75 ppm), Se considera normal 0.1 ppm y para los ríos amazónicos se considera optimo 0,02 ppm (Cleber y Alho, 2004). De acuerdo a los promedios de todos los puntos de la Tablas 5 y 6 , se observa en todos los elementos un ligero incremento en todos los puntos. 
Tabla 1. Límites máximos permisibles de metales en agua según norma peruana

\begin{tabular}{lccc}
\hline ELEMENTO & $\begin{array}{c}\text { CATEGORIA 1. AGUAS } \\
\text { RECREACIONALES. B1 } \\
\text { CONTACTO } \\
\text { PRIMARIO, ppm }\end{array}$ & $\begin{array}{c}\text { CATEGORIA 3. } \\
\text { AGUAS DE } \\
\text { RIEGO, ppm }\end{array}$ & $\begin{array}{c}\text { CATEGORIA 4. } \\
\text { CONSERVACION DEL } \\
\text { AMBIENTE ACUATICO } \\
\text { EN RIOS DE SELVA, } \\
\text { ppm }\end{array}$ \\
\hline $\mathrm{Cu}$ & 2 & 0,5 & 0,02 \\
$\mathrm{Fe}$ & 0,3 & 1,0 & - \\
$\mathrm{Mg}$ & - & 150 & - \\
$\mathrm{Zn}$ & 3 & 24 & 0,3 \\
$\mathrm{Mn}$ & 0,1 & 0,2 & - \\
$\mathrm{Pb}$ & 0,01 & 0,05 & 0,001 \\
$\mathrm{Cd}$ & 0,01 & 0,01 & 0,004 \\
$\mathrm{Hg}$ & 0,001 & 0,001 & 0,001 \\
\hline
\end{tabular}

Tabla 2. Puntos de muestreo en cuenca del rio nanay, 2011

\begin{tabular}{|c|c|c|c|c|c|}
\hline \multirow{2}{*}{ Punto de muestreo en creciente } & \multicolumn{2}{|c|}{ Coordenadas } & \multirow{2}{*}{ Procedencia } & \multirow[t]{2}{*}{$\mathrm{pH}$} & \multirow[t]{2}{*}{ Temp. ${ }^{\circ} \mathrm{C}$} \\
\hline & $\mathrm{S}$ & 0 & & & \\
\hline P 01 & $3050,705^{\prime}$ & $73^{\circ} 27,297^{\prime}$ & 200 m. Arriba Libertad & 4,7 & 27 \\
\hline P 02 & $3050,715^{\prime}$ & $73^{\circ} 27,282^{\prime}$ & 200 m Abajo Libertad & 4,5 & 27 \\
\hline P 03 & $3050,412^{\prime}$ & $73^{\circ} 23,299^{\prime}$ & Nina Rumi & 5,8 & 28 \\
\hline P 04 & $3045,099 '$ & $73^{\circ} 16,816^{\prime}$ & Pampachica & 4,6 & 28 \\
\hline P 05 & 3044,720 & $73^{\circ} 15,930^{\prime}$ & Morona & 5,3 & 29 \\
\hline P 06 & $3042,094^{\prime}$ & $73^{\circ} 15,087^{\prime}$ & Bellavista & 7,0 & 30 \\
\hline \multicolumn{6}{|l|}{ Punto de muestreo en vaciante } \\
\hline P 01 & $3050,705^{\prime}$ & $73^{\circ} 27,297^{\prime}$ & Arriba Libertad & 5,3 & 26,8 \\
\hline P 02 & $3050,715^{\prime}$ & $73^{\circ} 27,282^{\prime}$ & Abajo Libertad & 5,3 & 26,8 \\
\hline P 03 & $3050,412^{\prime}$ & $73^{\circ} 23,299^{\prime}$ & Nina Rumi & 4,8 & 27 \\
\hline P 04 & $3045,099 '$ & $73^{\circ} 16,816^{\prime}$ & Pampachica & 5,1 & 27 \\
\hline P 05 & $3043,385^{\prime}$ & $73^{\circ} 16,418$ & Munich & 6,6 & 29,5 \\
\hline P 06 & $3042,094^{\prime}$ & $73^{\circ} 15,087^{\prime}$ & Bellavista & 5,7 & 28 \\
\hline
\end{tabular}

Tabla 3. Metales pesados en cuenca del rio nanay en creciente del año 2011

\begin{tabular}{llcccc}
\hline $\begin{array}{c}\text { Puntos de } \\
\text { muestreo }\end{array}$ & \multicolumn{1}{c}{ Zona } & $\begin{array}{c}\mathrm{Cu} \\
\mathrm{ppm}\end{array}$ & $\begin{array}{c}\mathrm{Fe} \\
\mathrm{ppm}\end{array}$ & $\begin{array}{c}\mathrm{Mg} \\
\mathrm{ppm}\end{array}$ & $\begin{array}{c}\mathrm{Zn} \\
\mathrm{ppm}\end{array}$ \\
\hline P 01 & 200 m Arriba Libertad & $0,004 \pm 0,001^{\mathrm{a}}$ & $0,806 \pm 0,015^{\mathrm{a}}$ & $0,501 \pm 0,009 \mathrm{a}$ & $0,115 \pm 0,011 \mathrm{a}$ \\
P 02 & 200 m Abajo Libertad & $0,004^{\mathrm{a}}$ & $1,594 \pm 0,022 \mathrm{~b}$ & $0,276 \pm 0,002 \mathrm{~b}$ & $0,055 \mathrm{~b}$ \\
P 03 & Nina Rumi & $0,002 \mathrm{~b}$ & $0,922 \pm 0,049 \mathrm{c}$ & $0,241 \pm 0,014 \mathrm{c}$ & $0,050 \pm 0,001 \mathrm{c}$ \\
P 04 & Pampachica & $0,002 \mathrm{~b}$ & $1,015 \pm 0,021 \mathrm{~d}$ & $0,266 \pm 0,008 \mathrm{~d}$ & $0,049 \pm 0,001 \mathrm{c}$ \\
P 05 & Morona & $0,009 \pm 0,001 \mathrm{c}$ & $2,207 \pm 0,047 \mathrm{e}$ & $0,945 \pm 0,016 \mathrm{e}$ & $0,136 \pm 0,004 \mathrm{~d}$ \\
P 06 & Bellavista & $0,008 \mathrm{~d}$ & $1,108 \pm 0,039 \mathrm{f}$ & $0,324 \pm 0,018 \mathrm{f}$ & $0,040 \pm 0,001 \mathrm{e}$ \\
& PROMEDIO & 0,005 & 1,275 & 0,426 & 0,074 \\
& SD \pm & 0,0030 & 0,5311 & 0,2712 & 0,0406 \\
\hline \multicolumn{5}{c}{}
\end{tabular}

Continuación Tabla 3. 


\begin{tabular}{llcccc}
\hline $\begin{array}{c}\text { Puntos de } \\
\text { muestreo }\end{array}$ & \multicolumn{1}{c}{ Zona } & $\begin{array}{c}\mathrm{Mn} \\
\mathrm{ppm}\end{array}$ & $\begin{array}{c}\mathrm{Pb} \\
\mathrm{ppm}\end{array}$ & $\begin{array}{c}\mathrm{Cd} \\
\mathrm{ppm}\end{array}$ & $\begin{array}{c}\mathrm{Hg} \\
\mathrm{ppm}\end{array}$ \\
\hline P 01 & 200 m Arriba Libertad & $0,023 \pm 0,004 \mathrm{a}$ & $0,113 \pm 0,006 \mathrm{a}$ & $0,002 \mathrm{a}$ & $0,0003 \pm 0,0001 \mathrm{a}$ \\
P 02 & 200 m Abajo Libertad & $0,032 \pm 0,003 \mathrm{~b}$ & $0,083 \pm 0,006 \mathrm{~b}$ & $0,001 \mathrm{~b}$ & $0,0004 \pm 0,0001 \mathrm{a}$ \\
P 03 & Nina Rumi & $0,035 \pm 0,001 \mathrm{c}$ & $0,087 \pm 0,012 \mathrm{~b}$ & $0,001 \mathrm{~b}$ & $0,0003 \pm 0,0001 \mathrm{a}$ \\
P 04 & Pampachica & $0,045 \pm 0,004 \mathrm{~d}$ & $0,103 \pm 0,006 \mathrm{a}$ & $0,001 \mathrm{~b}$ & $0,0003 \pm 0,0001 \mathrm{a}$ \\
P 05 & Morona & $0,121 \pm 0,008 \mathrm{e}$ & $0,137 \pm 0,012 \mathrm{c}$ & $0,001 \mathrm{~b}$ & $0,0025 \pm 0,0001 \mathrm{a}$ \\
P 06 & Bellavista & $0,067 \pm 0,003 \mathrm{f}$ & $0,143 \pm 0,006 \mathrm{c}$ & $0,001 \mathrm{~b}$ & $0,0003 \pm 0,0002 \mathrm{~b}$ \\
& PROMEDIO & 0,054 & 0,111 & 0,001 & 0,0007 \\
& SD \pm & 0,0362 & 0,0250 & 0,0004 & 0,0009 \\
\hline
\end{tabular}

Nota: letras diferentes en la misma columna presentan diferencia significativa a $p<0,05$

Tabla 4. Metales pesados en agua de la cuenca del rio nanay en vaciante, 2011.

\begin{tabular}{llcccc}
\hline Cod. Lab. & Procedencia & $\begin{array}{c}\mathrm{Cu} \\
\text { ppm }\end{array}$ & $\begin{array}{c}\text { Fe } \\
\text { ppm }\end{array}$ & $\begin{array}{c}\text { Mg } \\
\text { ppm }\end{array}$ & $\begin{array}{c}\text { Zn } \\
\text { ppm }\end{array}$ \\
\hline P 01 & Arriba Libertad & $0,004 \pm 0,005 \mathrm{a}$ & $1,185 \pm 0,020 \mathrm{a}$ & $0,209 \pm 0,007 \mathrm{a}$ & $0,030 \pm 0,004 \mathrm{a}$ \\
P 02 & Abajo Libertad & $0,0044 \pm 0,002 \mathrm{a}$ & $1,588 \pm 0,112 \mathrm{c}$ & $0,275 \pm 0,013 \mathrm{c}$ & $0,068 \pm 0,011 \mathrm{~b}$ \\
P 03 & Nina Rumi & $0,002 \pm 0,007 \mathrm{~b}$ & $1,106 \pm 0,140 \mathrm{a}$ & $0,216 \pm 0,020 \mathrm{a}$ & $0,033 \pm 0,010 \mathrm{a}$ \\
P 04 & Pampachica & $0,002 \pm 0,003 \mathrm{~b}$ & $1,122 \pm 0,032 \mathrm{bc}$ & $0,2438 \pm 0,009 \mathrm{bc}$ & $0,038 \pm 0,009 \mathrm{a}$ \\
P 05 & Munich & $0,0087 \pm 0,008 \mathrm{c}$ & $1,696 \pm 0,092 \mathrm{~d}$ & $0,6739 \pm 0,036 \mathrm{~d}$ & $0,058 \pm 0,010 \mathrm{~b}$ \\
P 06 & Bellavista & $0,0075 \pm 0,004 \mathrm{c}$ & $1,008 \pm 0,094 \mathrm{ab}$ & $0,2273 \pm 0,013 \mathrm{ab}$ & $0,042 \pm 0,009 \mathrm{a}$ \\
& PROMEDIO & 0,005 & 1,284 & 0,308 & 0,045 \\
& SD \pm & 0,0028 & 0,2850 & 0,1810 & 0,0150 \\
\hline
\end{tabular}

Nota: letras diferentes en la misma columna presentan diferencia significativa a $p<0,05$

Continuación Tabla 4.

\begin{tabular}{llcccc}
\hline Cod. Lab. & Procedencia & $\begin{array}{c}\mathrm{Mn} \\
\mathrm{ppm}\end{array}$ & $\begin{array}{c}\mathrm{Pb} \\
\mathrm{ppm}\end{array}$ & $\begin{array}{c}\mathrm{Cd} \\
\mathrm{ppm}\end{array}$ & $\begin{array}{c}\mathrm{Hg} \\
\mathrm{ppm}\end{array}$ \\
\hline P 01 & Arriba Libertad & $0,015 \pm 0,005$ & $0,005 \mathrm{a}$ & $0,002 \mathrm{a}$ & $0,0020 \pm 0,0002 \mathrm{a}$ \\
P 02 & Abajo Libertad & $0,013 \pm 0,001$ & $0,018 \pm 0,003 \mathrm{a}$ & $0,001 \mathrm{~b}$ & $0,0040 \pm 0,0001 \mathrm{~b}$ \\
P 03 & Nina Rumi & $0,008 \pm 0,002$ & $0,072 \pm 0,006 \mathrm{~b}$ & $0,001 \mathrm{~b}$ & $0,0240 \pm 0,0001 \mathrm{a}$ \\
P 04 & Pampachica & $0,009 \pm 0,003$ & $0,074 \pm 0,010 \mathrm{~b}$ & $0,001 \mathrm{~b}$ & $0,0090 \pm 0,0001 \mathrm{a}$ \\
P 05 & Munich & $0,013 \pm 0,002$ & $0,005 \mathrm{a}$ & $0,001 \mathrm{~b}$ & $0,0070 \pm 0,0001 \mathrm{a}$ \\
P 06 & Bellavista & $0,008 \pm 0,008$ & $0,092 \pm 0,007 \mathrm{c}$ & $0,001 \mathrm{~b}$ & $0,0040 \pm 0,0001 \mathrm{~b}$ \\
& PROMEDIO & 0,011 & 0,053 & 0,001 & 0,008 \\
& SD \pm & 0,0030 & 0,0376 & 0,0004 & 0,0081 \\
\hline
\end{tabular}

Nota: letras diferentes en la misma columna presentan diferencia significativa a $p<0,05$ 
Tabla 5. Metales pesados en sedimentos en cuenca del rio nanay en creciente, 2011.

\begin{tabular}{cccccccc}
\hline \multirow{2}{*}{ Pts } & \multirow{2}{*}{ Zona } & \multicolumn{3}{c}{ Zn } & \multicolumn{2}{c}{ Cd } \\
\cline { 3 - 7 } & & ppm & SD & ppm & SD & ppm & SD \\
\hline 1 & Arriba Libertad & $26,50 \mathrm{a}$ & 0,56 & $48,48 \mathrm{a}$ & 0,10 & $0,28 \mathrm{a}$ & 0,04 \\
2 & Abajo Libertad & $51,47 \mathrm{~b}$ & 1,17 & $68,62 \mathrm{~b}$ & 0,06 & $0,18 \mathrm{~b}$ & 0,02 \\
3 & Nina Rumi & $39,13 \mathrm{c}$ & 1,21 & $39,82 \mathrm{c}$ & 0,02 & $0,11 \mathrm{c}$ & 0,03 \\
4 & Pampachica & $65,83 \mathrm{~d}$ & 0,74 & $70,70 \mathrm{~d}$ & 0,06 & $0,12 \mathrm{bc}$ & 0,01 \\
5 & Morona & $43,67 \mathrm{e}$ & 0,15 & $105,32 \mathrm{e}$ & 0,22 & $0,44 \mathrm{~d}$ & 0,07 \\
6 & Bellavista & $16,30 \mathrm{f}$ & 0,61 & $34,58 \mathrm{f}$ & 0,05 & $0,34 \mathrm{a}$ & 0,01 \\
& Prom., ppm & 40,48 & & 61,25 & & 0,24 & \\
& SD & 17,64 & & 26,15 & & 0,13 & \\
\hline
\end{tabular}

Nota: letras diferentes en la misma columna presentan diferencia significativa a $p<0,05$

Continuación Tabla 5.

\begin{tabular}{cccccccc}
\hline \multirow{2}{*}{ Pts } & \multirow{2}{*}{ Zona } & \multicolumn{2}{c}{ Fe } & \multicolumn{2}{c}{ Pb } & \multicolumn{2}{c}{ Hg } \\
\cline { 3 - 7 } & & ppm & SD & ppm & SD & ppm & SD \\
\hline 1 & Arriba Libertad & $2747,62 \mathrm{a}$ & 3,05 & 18,63 & 1,90 & $0,942 \mathrm{a}$ & 0,033 \\
2 & Abajo Libertad & $3212,67 \mathrm{~b}$ & 3,40 & 72,53 & 1,17 & $1,205 \mathrm{~b}$ & 0,038 \\
3 & Nina Rumi & $857,68 \mathrm{c}$ & 6,96 & 56,07 & 0,38 & $1,172 \mathrm{~b}$ & 0,038 \\
4 & Pampachica & $2505,07 \mathrm{~d}$ & 2,33 & 68,33 & 0,72 & $2,557 \mathrm{c}$ & 0,016 \\
5 & Morona & $2500,89 \mathrm{~d}$ & 3,99 & 55,21 & 0,59 & $2,025 \mathrm{~d}$ & 0,015 \\
6 & Bellavista & $979,53 \mathrm{e}$ & 2,59 & 20,78 & 0,31 & $1,913 \mathrm{~d}$ & 0,075 \\
& Prom., ppm & 2133,91 & & 48,59 & & 1,636 & \\
& SD & 977,15 & & 23,38 & & 0,022 & \\
\hline
\end{tabular}

Nota: letras diferentes en la misma columna presentan diferencia significativa a $p<0,05$

Tabla 6. Metales pesados en sedimentos en cuenca del rio nanay en vaciante, 2011.

\begin{tabular}{|c|c|c|c|c|c|c|c|c|c|c|c|c|c|}
\hline \multirow{2}{*}{ Pts } & \multirow{2}{*}{ Zona } & \multicolumn{2}{|c|}{$\mathrm{Cu}$} & \multicolumn{2}{|l|}{ Zn } & \multicolumn{2}{|c|}{ Cd } & \multicolumn{2}{|l|}{$\mathbf{F e}$} & \multicolumn{2}{|c|}{$\mathbf{P b}$} & \multicolumn{2}{|c|}{$\mathrm{Hg}$} \\
\hline & & ppm & SD & ppm & SD & ppm & SD & ppm & SD & ppm & SD & ppm & SD \\
\hline \multirow[t]{3}{*}{1} & Arriba & & & & & & & & & & & & \\
\hline & Libertad & $26,13^{a}$ & 1,66 & $50,02 a$ & 0,50 & $0,47 a$ & 0,01 & $2776,11 \mathrm{a}$ & 3,05 & $22,12 \mathrm{a}$ & 0,41 & $1,942 a$ & 0,033 \\
\hline & Libertad & $53,27 \mathrm{~b}$ & 1,10 & $71,65 b$ & 0,99 & $0,30 c$ & 0,02 & $3241,16 b$ & 3,40 & $40 \mathrm{~b}$ & 0,61 & & 0,081 \\
\hline 3 & Nina Rumi & $45,43 v$ & 0,57 & $44,09 c$ & 0,45 & $0,14 b$ & 0,01 & $1142,58 \mathrm{c}$ & 6,96 & $51,10 \mathrm{c}$ & 0,21 & $2,845 c$ & 0,068 \\
\hline 4 & Pampachica & $70,27 d$ & 0,50 & $77,36 d$ & 0,76 & $0,14 b$ & 0,02 & $2789,97 a$ & 2,33 & $72,95 d$ & 0,41 & $4,062 d$ & 0,046 \\
\hline 5 & Munich & $48,83 e$ & 0,31 & $114,10 \mathrm{e}$ & 0,15 & $0,54 d$ & 0,01 & $2785,79 a$ & 3,99 & $60,39 e$ & 0,58 & $3,673 e$ & 0,051 \\
\hline \multirow[t]{3}{*}{6} & Bellavista & $17,90 f$ & 0,56 & $41,25 f$ & 0,35 & $0,47 a$ & 0,01 & $1273,92 d$ & 18,20 & $25,41 f$ & 0,44 & $3,417 f$ & 0,049 \\
\hline & Prom., ppm & 43,64 & & 66,41 & & 0,34 & & 2334,92 & & 51,40 & & 3,003 & \\
\hline & SD & 17,32 & 0,46 & 25,24 & 0,27 & 0,16 & 0,00 & 813,80 & 5,51 & 21,23 & 0,13 & 0,055 & \\
\hline
\end{tabular}




\section{CONCLUSIONES}

Es importante reconocer que aun se mantienen altas concentraciones de plomo y mercurio en las aguas del río Nanay, por lo cual se debe de considerar este punto para mejorar el tratamiento de este líquido vital, por ser este río fuente de abastecimiento de la ciudad de Iquitos. Se debe de considerar que los sedimentos presentan contaminación de metales pesados, específicamente alta de mercurio y que deja el río en época de vaciante, presenta sus riesgos para la salud humana, debido a que en estos lugares, se practica la agricultura.

\section{REFERENCIAS}

Adam P. 1990. Saltmarsh ecology. Cambridge University Press. Cambridge. Gran Bretaña. $461 \mathrm{p}$

Alloway BJ. (1995): Heavy metals in soils. B.J. Alloway (ed.). $2^{a}$ ed. Blackie Academic \& Professional.370 p.

Álvarez J, Sotero V, Ipenza C, Brack, A. 2011. Minería aurífera en madre de Dios y contaminación con mercurio- IIAPMINAM. Lima. Ed. Súper Gráfica. 103p.

Avila-Pérez $P$, Balcázar $M$, Zarazúa-Ortega $G$, Barceló-Quintal I, Díaz-Delgado C. 1999. Heavy metal concentrations in water and bottom sediments of a Mexican reservoir. The Science of the Total Environment 234, 185-196.

Barreto L. 2003. Contaminación en ka cuenca del rio Nanay por efecto de la actividad minera. Ambiente ecológico. Edicion 86.

http://www.ambientecologico.com/edicio nes/2004/088 01.2004/088 Sumario Inv estigacion.php3

[Consultado Octubre 2011]

Baryshev VB, Golubev AV, Dvurechenskaya SY, Sokolovskaya IP, Trunova VA. 1995. Multielemental analysis of Novosibirsk reservoirs and some of its tributaries bottom sediments by the SRXFA technique. Nucl. Instrument. Methods Phys. Res. 359, 305-306.

Brannon JM, Plumb RH \& Smith I. 1980. Longterm release of heavy metals from sediments. En R.A. Baker (ed.) Contaminants and sediments. Vol 2.
Analysis, chemistry, biology. An Arbor

Science, MI, pp. 221-266.

Burgess RM \& Scott KJ. 1992. The significance of inplace contaminated marine sediments on the water column: Process and effects. En G.A. Burton (ed.), Sediment Toxicity Assesment. Lewis Publishers, Chelsea, MI, pp. 129-165.

Caldas ED, Machado LL. 2004. Cadmiun, mercury and lead in medicinal herbs in Brazil. Food Chem Toxicol 42(4), 599-603.

Cleber L, Alho J. 2004. Contaminação por Mercúrio em Sedimento e Moluscos da Bacia do rio Bento Gomes, MT. Boletim de pesquisa e desenvolvimento, EMBRAPA. 24p.

García J, Diaz C, Quentin E, Ávila P, Tejada S, Zarzua G. 2007. Caracterización de la contaminación por metales pesados y reducción de la capacidad de almacenamiento hidráulico por azolve de un embalse mexicano. Hidrobiologica. $17(2), 127-130$.

Gómez R. 1995. Diagnóstico sobre la contaminación ambiental en la Amazonia. Documento Técnico No 15 . IIAP. Iquitos. $25 \mathrm{p}$.

IIAP (Instituto de Investigaciones de la Amazonía Peruana), Pontificia Universidad Católica del Perú, Foro Peruano para el Agua. 2009. Diagnóstico y marco estratégico para la gestión integrada de la cuenca del río Nanay, Loreto. Documento en consulta.

IIAP. 2002. Propuesta De zonificacion ecologica economica de la cuenca del rio nanya, volumenII. Medio físico. Proeycto conservación de la biodiversidad y manejo comunitario de los recursos naturales de la cuenca del rio Nanay, 118p.

IIAP. 2008. Evaluación de metales pesados procedentes del botadero municipal en el año 2008. Instituto de Investigaciones de la Amazonia Peruana. Informe técnico. 14p.

Janiot L, Sik E, Marcucci O, Gesino A, Molina D, Martinez L, Marcuco P. 2001. Contaminantes orgânicos persistentes (COPs) y metales pesados em água $y$ sedimentos.del rio de la Plata y su frente 
marítimos. Servicio Hifrografia naval, Buenos Aires. 4p.

Jorheim L. 2000. Determination of metals in food by atomic absortion spectrometry alter dry ashing: NMKL collaborative study. Journal of AOAC Internacional. 83(5), 1204-11.

Labunska I, Brigden K, Stronger R. 2000. Contaminantes orgánicos y metales pesados en sedimentos y muestras de agua asociada. INFORME. Greenpeace..Laboratorio de Investigación de Grenpaece, Departamento de ciencias Biológicas. Universidad de Exeter. Reino Unido. 14p.

Linsley R, Kohler M, Paulhus J. 1994. Hidrologia para ingenieros.Mc Graw-Hill. México. 331-345.

López M. 2009. Distribución y fitodisponibilidad de metales pesados (sb, hg, as) en los jales de la mina de antimonio de wadley, estado de San Luis Potosí. Tesis de Maestria. Programa de Posgraduacion en Ciencias de la Tierra. UNAM. Mexico. 160p.

Mexico. 1994. Norma Oficial Mexicana NOM 117-1994. Bienes y servicios. Método de prueba para la determinación de cadmio, arsénico, plomo, estaño, cobre, fierro, zinc y mercurio en alimentos, agua potable y agua purificada por espectrometría de absorción atómica. $10 \mathrm{p}$.

Monroy M, Diaz F, Dazo L, Carrizales L. 2002. Evaluación de la contameincion por arsénico y metales pesados $(\mathrm{Pb}, \mathrm{Cu}, \mathrm{Zn})$ y análisis de riesgo en salud en villa de la
Paz-Matehuale - SLP. Nota informativaFac de Ingenieria. Instituto de Metalurgia. UASLP.-San Luis de Potosí. 7p.

Perú. 2008. Ministerio del ambiente. Estándares nacionales de calidad ambiental para agua D.S. No 002-2008MINAM. El Peruano. Lima. Julio31, 2008. P. 37722-377227.

Rodriguez S y Avila-Pérez P. 1997. Stock of immobilized metals in "José Antonio Alzate" dam mud sediment samples in the State of México, México. J Radioanal Nuc Chem 221, 231-234.

Rojas R. 1986. Contaminación del rio Rimac por metales pesados provenientes de las industrias mineras. Reunión Interamericana sobre el control de vertimientos de sustancias tóxicas. Sao Paulo. Brasil. 17-21 marzo.76p.

Sánchez $F$, Márquez $C$, Juárez M, Martínez J, Miranda P, Esquivel C, Juarez M. 2000. Análisis de metales pesados en las aguas residuales del rio San Javier y repercusión en la salud e impacto ambiental. Documento Técnico. Departamento de Química. Unidad Interdisciplinar de Biotecnología. IPNB. México. 4p.

Valdés F, Cabrera VM. 1999. La contaminación por metales pesados en Torreón, Coahuila, México. $1^{\text {a }}$ Ed. Texas Center for Policy Studies, CILADHAC, Torreón, Coah.

Von Gunten HR, Sturm M, Moser RN. 1997. 200-Year record of metals in lake sediments and natural background concentrations. Environ Sci Technol 31, 2193-2197. 